\title{
A Comparison of Sufficiency Condtions for the Goldbach and the Twin Primes Conjectures
}

\author{
C. J. Mozzochi \\ Princeton, NJ, USA \\ Email: cjm@ix.netcom.com
}

Received 6 February 2014; revised 6 March 2014; accepted 15 March 2014

Copyright (C) 2014 by author and Scientific Research Publishing Inc.

This work is licensed under the Creative Commons Attribution International License (CC BY).

http://creativecommons.org/licenses/by/4.0/

\section{c) (i) Open Access}

\section{Abstract}

It is generally known that under the generalized Riemann hypothesis one could establish the twin primes conjecture by the circle method, provided one could obtain the estimate $o\left(n \log ^{-2} n\right)$ for the integral of the representation function over the minor arcs. One of the new results here is that the assumption of GRH can be removed. We compare this and other such sufficiency results with similar results for the Goldbach conjecture.

\section{Keywords}

Goldbach Conjecture, Twin Primes Conjecture, Circle Method, Generalized Riemann Hypothesis

\section{Introduction}

Let $n \geq n_{0}$ and $\log ^{15} n \leq P \leq n^{\epsilon}$ for $\epsilon>0$. Let $x_{0}=x_{0}(n) \rightarrow 0$ rapidly. When $0<h \leq q \leq P$ and $(h, q)=1$ let $M(h, q)$ denote the closed interval $\left[\frac{h}{q}-x_{o}, \frac{h}{q}+x_{0}\right]$, a so-called major arc.

It is easily shown, for any choice of $P$, that all the $M(h, q)$ are disjoint and contained in the closed interval $\left[x_{0}, 1+x_{0}\right]$.

For each $n$ let $m(n)$ be those points in $\left[x_{0}, 1+x_{0}\right]$ which are not in any closed neighborhood (major arc) of radius $x_{0}$ about any rational number $\frac{h}{q}$, where $(h, q)=1$ and $q \leq P$.

For each $n$ let $m^{*}(n)$ be those points in $\left[x_{0}, 1+x_{0}\right]$ which are not in any closed neighborhood (major arc) 
of radius $x_{0}$ about any rational number $\frac{h}{q}$, where $(h, q)=1,(q, n)=1$ and $q \leq \log ^{15} n$.

Let $r(n)$ denote the number of ways $n$ (even) can be represented as a sum of two primes.

Let $r^{\prime}(n)$ denote the number of twin primes less than or equal to $n$.

$$
\begin{gathered}
\text { Let } f(x, v)=\sum_{p \leq v} \epsilon(p x) \text { where } \epsilon(z)=\epsilon^{2 \pi \mathrm{iz}}(v \geq 0) . \\
\text { Let } g(x, v)=\sum_{2 \leq m \leq v} \frac{\epsilon(m x)}{\log m}(v \geq 2), g(x, v)=0(v<2) . \\
L s[v]=\sum_{2 \leq m \leq v} \frac{1}{\log m}=g(0, v) \sim\left(\frac{v}{\log v}\right), L s[0]=L s[1]=0 .
\end{gathered}
$$

In [1] the following two theorems are established:
Theorem 1.1 Under the generalized Riemann hypothesis with $P=n^{\epsilon}$ and $x_{0}=\frac{\log _{n}^{15}}{n}$ if

$$
\int_{m(n)} f^{2}(x, n) \epsilon(-n x) \mathrm{d} x=0\left(n \log ^{-2} n\right) \text {, then } r(n)>0 \text { for all even } n \geq n_{0} .
$$

Theorem 1.2 Let $P=\log ^{15} n$ and $x_{0}=\frac{\log ^{15} n}{n}$ if

$$
\int_{m^{*}(n)} f^{2}(x, n) \epsilon(-n x) \mathrm{d} x=0\left(n \log ^{-2} n\right) \text {, then } r(n)>0 \text { for all even } n \geq n_{0} .
$$

In [2] the following two theorems are established:

Theorem 1.3 Under the assumption that Siegel zeros do not exist with $P=\exp \left(c \log ^{1 / 2} n\right)$ and $x_{0}=\frac{P}{n}$ if

$$
\int_{m(n)} f^{2}(x, n) \epsilon(-n x) \mathrm{d} x=o\left(n \log ^{-2} n\right) \text {, then } r(n)>0 \text { for all even } n \geq n_{0} \text {. }
$$

Theorem 1.4 Let $P=\exp \left(c \log ^{1 / 2} n\right)$ and $x_{0}=\frac{P}{n}$. If

$$
\int_{m(n)} f^{2}(x, n) \epsilon(-n x) \mathrm{d} x=o\left(n P^{-1 / 32} \log ^{-2} n\right) \text {, then } r(n)>0 \text { for all even } n \geq n_{0} \text {. }
$$

The proof of Theorem 1.3 and, in particular, the proof of Theorem 1.4 is very complicated.

In Section 6 of [2] it is shown, by a very complicated argument, that a particularly natural approach for eliminating the condition $(q, n)=1$ in Theorem 1.2 does not work.

As we will now see, the situation with regard to the twin prime conjecture is significantly, very less complicated. The reason is primarily because we only need to consider the Ramanujan sums $C_{q}(2)$ rather than the sums $C_{q}(n)$, which appear in all of the theorems above, related to the Goldbach conjecture.

In Section 2 we will establish $n$ Theorem 1.5 Let $P=\log ^{15} n$ and $x_{0}=\frac{\log ^{15} n}{n} \cdot r^{\prime}(n) \rightarrow \infty$ if $\int_{m(n)}|f(x, n)|^{2} \epsilon(-2 x) \mathrm{d} x=o\left(n \log ^{-2} n\right)$ as

\section{A Proof of Theorem $\mathbf{1 . 5}$}

$$
r^{\prime}(n)=\int_{x_{0}}^{x_{0}+1}|f(x, n)|^{2} \epsilon(-2 x) \mathrm{d} x
$$

We decompose the above integral

$$
\begin{gathered}
r^{\prime}(n)=\int_{m(n)}|f(x, n)|^{2} \epsilon(-2 x) \mathrm{d} x+\int_{M(n)}|f(x, n)|^{2} \epsilon(-2 x) \mathrm{d} x . \\
r^{\prime}(n)=A(n)+B(n) .
\end{gathered}
$$

It is immediate by the prime numbers theorem that 


$$
A(n)=O\left(n \log ^{-1} n\right)
$$

By definition

$$
\int_{M(n)}|f(x, n)|^{2} \epsilon(-2 x) \mathrm{d} x=\sum_{q \leq \log ^{15} n} \sum_{\substack{0<h \leq q \\(h, q)=1}} T(h, q)
$$

where

$$
T(h, q)=\int_{\frac{h}{q}-x_{0}}^{\frac{h}{q}+x_{0}}|f(x, n)|^{2} \epsilon(-2 x) \mathrm{d} x .
$$

Lemma 2.1 Let $q \leq \log ^{15} n,|y| \leq x_{0},(h, q)=1$ and $n \geq n_{0}$. Then

$$
\left|f\left(\frac{h}{q}+y, n\right)-\frac{u(q)}{\phi(q)} g(y, n)\right| \leq n \log ^{-69} n
$$

This is Theorem 58 in [3].

Lemma 2.2 Under the hypothesis of Lemma 2.1 we have

$$
\left|f^{2}\left(\frac{h}{q}+y, n\right)-\frac{u^{2}(q)}{\phi^{2}(q)} g^{2}(y, n)\right| \leq C_{1} n^{2} \log ^{-69} n .
$$

Proof. This follows immediately from Lemma 2.1 and the trivial inequalities $|f(x, n)| \leq n$ and $|g(y, n)| \leq n$ and the fact that if $|a| \leq n$ and $b \leq n$, then $\left|a^{2}-b^{2}\right| \leq 2 n|a-b|$.

Hence it is immediate that

By the change of variable $y=\left(x-\frac{h}{q}\right)$ we have

$$
\left.|| f\left(\frac{h}{q}+y, n\right)\right|^{2}-\frac{u^{2}(q)}{\phi^{2}(q)}|g(y, n)|^{2} \mid \leq C_{1} n^{2} \log ^{-69} n .
$$

$$
T(h, q)=\in\left(-\frac{2 h}{q}\right) \int_{-x_{0}}^{x_{0}}\left|f\left(\frac{h}{q}+y, n\right)\right|^{2} \epsilon(-2 y) \mathrm{d} y .
$$

However, by (2.1) we have

$$
\begin{aligned}
& \left.\left|\epsilon\left(-\frac{2 h}{q}\right) \int_{-x_{0}}^{x_{0}}\right| f\left(\frac{h}{q}+y, n\right)\right|^{2} \epsilon(-2 y) \mathrm{d} y-\frac{u^{2}(q)}{\phi^{2}(q)} \epsilon\left(-2 \frac{h}{q}\right)_{-x_{0}}^{x_{0}}|g(y, n)|^{2} \epsilon(-2 y) \mathrm{d} y \mid \\
& \leq\left.\int_{-x_{0}}^{x_{0}}|| f\left(\frac{h}{q}+y, n\right)\right|^{2}-\frac{u^{2}(q)}{\phi^{2}(q)}|g(y, n)|^{2} \mid \mathrm{d} y \leq \int_{-x_{0}}^{x_{0}} C_{1} n^{2} \log ^{-69} n \mathrm{~d} y \\
& =2 x_{0} C_{1} n^{2} \log ^{-69} n=C_{2} n \log ^{-54} n .
\end{aligned}
$$

Let $T_{1}(n)=\int_{-x_{0}}^{x_{0}}\left|g^{2}(y, n)\right|^{2} \epsilon(-2 y)$ d $y$ so that if $(h, q)=1, q \leq \log ^{15} n$, then

$$
\left|T(h, q)-\frac{u^{2}(q)}{\phi^{2}(q)} T_{1}(n) \epsilon\left(-\frac{2 h}{q}\right)\right| \leq C_{2} n \log ^{-54} n .
$$

Let $T(n)=\sum_{m_{1} m_{2}} \log ^{-1} m_{1}, \log ^{-1} m_{2}$ with the condition of summation $n \geq m_{1} \geq 2, n \geq m_{2} \geq 2$ and $m_{2}-m_{1}=2$. It is easy to see that

$$
T(n)=\int_{-\frac{1}{2}}^{\frac{1}{2}}|q(y, n)|^{2} \epsilon(-2 y) \text { d } y \text { and } \frac{1}{3} n \log ^{-2} n \leq T(n) \leq n .
$$


For $(h, q)=1, q \leq \log ^{15} n$ we have

$$
\left|\epsilon\left(-\frac{2 h}{q}\right)\right|\left|\frac{u^{2}(q)}{u^{2}(q)}\right|\left|T(n)-T_{1}(n)\right| \leq \frac{1}{\phi^{2}(q)}\left(2 n \log ^{-15} n\right) .
$$

Hence for $(h, q)=1$ and $q \leq \log ^{15} n$

$$
\left|(h, q)-\frac{u^{2}(q)}{\phi^{2}(q)} T(n) \epsilon\left(-\frac{2 h}{q}\right)\right| \leq C_{2} n \log ^{-54} n+\frac{1}{\phi^{2}(q)}\left(2 n \log ^{-15} n\right) ;
$$

so that for some fixed $q \leq \log ^{15} n$ we have:

$$
\left|\sum_{\substack{0<h \leq q \\(h, q)=1}} T(h, q)-\frac{u^{2}(q)}{\phi^{2}(q)} T(n) \sum_{\substack{0<h \leq q \\(h, q)=1}} \epsilon\left(-\frac{2 h}{q}\right)\right| \leq\left(c_{2} n \log ^{-54} n\right) \phi(q)+\frac{1}{\phi^{4 / 3}(q)}\left(2 n \log ^{-15} n\right) \phi^{1 / 3}(q) .
$$

But $\phi(q) \leq \log ^{15} n$ and by definition

$$
\sum_{\substack{0<h \leq \leq \leq \\(h, q)=1}} \epsilon\left(-\frac{2 h}{q}\right)=C_{q}(2)
$$

so that it follows immediately that

$$
\left|\sum_{\substack{0<h \leq q \\(h, q)=1}} T(h, q)-\frac{u^{2}(q)}{\phi^{2}(q)} T(n) c_{q}(2)\right| \leq C_{2} n \log ^{-39} n+\frac{1}{\phi^{4 / 3}(q)}\left(2 n \log ^{-10} n\right) .
$$

Now summing over all $q \leq \log ^{15} n$ we have

$$
\begin{aligned}
\left|\sum_{q \leq \log ^{15}} \sum_{n<h \leq q)=1} T(h, q)-T(n) \sum_{q \leq \log ^{15} n} \frac{u^{2}(q)}{\phi^{2}(q)} C_{q}(2)\right| & \leq\left(C_{2} n \log ^{-39} n\right)\left(\log ^{15} n\right)+\sum_{q<\log ^{15} n} \frac{1}{\phi^{4 / 3}(q)}\left(2 n \log ^{-10} n\right) \\
& \leq C_{2} n \log ^{-24} n+C_{3}\left(2 n \log ^{-10} n\right) \leq C_{4} n \log ^{-10} n .
\end{aligned}
$$

since by Theorem 327 page 267 in [3]

$$
\sum_{q \leq \log ^{15} n} \frac{1}{\phi^{4 / 3}(q)} \leq C_{3}\left(C_{3} \text { independentof } n\right) .
$$

Hence

$$
\left|r^{\prime}(n)-T(n) \sum_{q \leq \log ^{15} n} \frac{u^{2}(q)}{\phi^{2}(q)} C_{q}(2)\right| \leq k(n) n \log ^{-2} n+C_{4} n \log ^{-10} n,
$$

where $k(n) \rightarrow 0$. Now let

$$
\begin{gathered}
R(n)=\sum_{q \leq \log ^{15} n} \frac{u^{2}(q)}{\phi^{2}(q)} C_{q}(2) \\
S=\sum_{q=1}^{\infty} \frac{u^{2}(q)}{\phi^{2}(q)} C_{q}(2)
\end{gathered}
$$

Lemma 2.3

$$
\sum_{q=1}^{\infty} \frac{u^{2}(q)}{\phi^{2}(q)} C_{q}(n)-\sum_{q \leq y} \frac{u^{2}(q)}{\phi^{2}(q)} C_{q}(n)=0\left(d(n)(\log \log 3 y)^{2} y^{-1}\right) .
$$

Proof. [4] page 211.

It is immediate by Lemma 2.3 that

$$
S-R(n)=0(1)
$$


What remains to be done is to show $S$ is bounded away from 0 .

Let

$$
f(q)=\frac{u(q)}{\phi(q)} C_{q}(2) .
$$

Since $u(q), \phi(q)$ and $C_{q}(2)$ are all multiplicative functions of $q, f$ is a multiplicative function of $q$. Also, by means of the trivial estimate on $C_{q}(2)$, namely 2, and a direct application of Theorem 327 page 267 in [5] we have

$$
\sum_{q=1}^{\infty}|f(q)| \leq 2 \sum_{q=1}^{\infty} \frac{1}{\phi^{2}(q)}<\infty ;
$$

so that by Theorem 2 [3] page 3 we have

$$
\begin{gathered}
S=\prod_{p} \sum_{m=0}^{\infty} f\left(p^{m}\right) \\
m=0 \quad f\left(p^{0}\right)=f(1)=1 \\
m=1 \quad f(p)=\frac{u^{2}(p)}{\phi^{2}(p)} C_{p}(2)=\frac{1}{(p-1)^{2}} C_{p}(2) \\
m \geq 2 \quad f(p)=0
\end{gathered}
$$

Hence

$$
S=\prod_{p}\left(1+\frac{1}{(p-1)^{2}} C_{p}(2)\right)=2 \underset{p>2}{ }\left(1-\frac{1}{(p-1)^{2}}\right)>2 \prod_{m=2}^{\infty}\left(1-\frac{1}{m^{2}}\right)=1 .
$$

\section{A Primitive Formulation of the Circle Method}

\section{1) Part I}

We assume $n$ (even) $>C_{0} . x_{0}=\left(\frac{\log ^{2+\epsilon} n}{n}\right)$.
For each $n$ let $E_{n}=\left[x_{0}, 1-x_{0}\right]$.

Let $r(n)$ be the number of representations of $n$ as the sum of two primes, each of which is less than $n$. Clearly,

$$
r(n)=\int_{x_{0}}^{1+x_{0}} f^{2}(x, n) \epsilon(-n x) \mathrm{d} x \text {, for each even } n .
$$

We decompose this integral

$$
r(n)=m(n)+T(1,1)
$$

where

$$
m(n)=\int_{E_{n}} f^{2}(x, n) \epsilon(-n x) \mathrm{d} x, T(1,1)=\int_{1-x_{0}}^{1+x_{0}} f^{2}(x, n) \epsilon(-n x) \mathrm{d} x .
$$

Clearly, by Theorem 55 in [3] and the last paragraph on page 63 in [3] we have

$$
|f(1, v)-g(0, v)| \leq n \log ^{-100} n \text {, uniformly }(0 \leq v \leq n) .
$$

By direct application of the easily established Equation (151) in [3]

$$
f\left(x_{1}+x_{2}, v\right)=\epsilon\left(v x_{2}\right) f\left(x_{1}, v\right)-2 \pi \mathrm{i} x_{2} \int_{0}^{v} \epsilon\left(u x_{2}\right) f\left(x_{1}, u\right) \mathrm{d} u,
$$

and the Equation (204) in [3] 


$$
g\left(x_{1}+x_{2}, v\right)=\epsilon\left(v x_{2}\right) g\left(x_{1}, v\right)-2 \pi \mathrm{i} x_{2} \int_{0}^{v} \epsilon\left(u x_{2}\right) g\left(x_{1}, u\right) \mathrm{d} u
$$

We have for $|y| \leq x_{0}$

$$
\begin{aligned}
& |f(1+y, n)-g(y, n)| \leq\left(n \log ^{-100} n\right) \\
& \left(1+2 \pi x_{0} n\right) \leq C_{1} n \log ^{-98+\varepsilon} n .
\end{aligned}
$$

By the trivial inequalities $|f(x, n)| \leq n$ and $|g(y, n)| \leq n$ and the fact that if $|a| \leq n$ and $|b| \leq n$, then $\left|a^{2}-b^{2}\right| \leq 2 n|a-b|$ with $a=f(1+y, n)$ and $b=g(y, n)$, we have for $|y| \leq x_{0}$

$$
\left|f^{2}(1+y, n)-g^{2}(y, n)\right| \leq C_{2} n^{2} \log ^{-98+\epsilon} n .
$$

By the change of variable $y=(x-1)$, we have

$$
T(1,1)=\int_{-x_{0}}^{x_{0}} f^{2}(1+y, n) \epsilon(-n y) \mathrm{d} y
$$

so that

$$
\begin{gathered}
\left|\int_{-x_{0}}^{x_{0}} f^{2}(1+y, n) \epsilon(-n y) \mathrm{d} y-\int_{-x_{0}}^{x_{0}} g^{2}(y, n) \epsilon(-n y) \mathrm{d} y\right| \leq \int_{-x_{0}}^{x_{0}}\left|f^{2}(1+y, n)-g^{2}(y, n)\right| \mathrm{d} y \leq C_{3} n \log ^{-96+2 \epsilon} n . \\
\text { Let } T_{1}(n)=\int_{-x_{0}}^{x_{0}} g^{2}(y, n) \epsilon(-n y) \mathrm{d} y \\
\left|T(1,1)-T_{1}(n)\right| \leq C_{3} n \log ^{-96+2 \epsilon} n \\
\text { Let } T(n)=\sum_{\substack{m_{1}, m_{2} \\
m_{1} \geq 2, m_{2} \geq 2}} \log ^{-1} m_{1} \log ^{-1} m_{2} .
\end{gathered}
$$

Clearly

$$
T(n)=\int_{-\frac{1}{2}}^{\frac{1}{2}} g^{2}(y, n) \epsilon(-n y) \mathrm{d} y .
$$

Also, the number of terms on the right hand side of (3.4) is $(n-3)$ and each term is greater than $\log ^{-2} n$ and less than 1 so that

$$
\begin{gathered}
\frac{1}{3} n \log ^{-2} n<T(n)<n . \\
\left|\sum_{m=2}^{m_{1}} \epsilon(m, y)\right| \leq \frac{1}{|\sin \pi y|} \leq \frac{1}{2|y|} ;\left(m_{1} \geq 2,0<|y| \leq \frac{1}{2}\right) .
\end{gathered}
$$

Hence by definition of $g(y, n)$ and Abel's lemma we have

$$
|g(y, n)|<|y|^{-1}, \quad\left(0<|y| \leq \frac{1}{2}\right)
$$

so that

$$
\left|T(n)-T_{1}(n)\right| \leq 2 \int_{x_{0}}^{\frac{1}{2}} y^{-2} \mathrm{~d} y \leq C_{4} x_{0}^{-1}=C_{4} n \log ^{-(2+\epsilon)} n .
$$

Hence, 


$$
|T(1,1)-T(n)| \leq C_{3} n \log ^{-96+2 \epsilon} n+C_{4} n \log ^{-(2+\epsilon)} n .
$$

Let $S(n)=1$. Then $|T(n) S(n)-T(n)|=0$

so that

$$
|r(n)-T(n) S(n)| \leq C_{5} n \log ^{-(2+\epsilon)} n+|m(n)|
$$

so that we have

$$
r(n)>0 \text { if } m(n)=o\left(n \log ^{-2} n\right) \text { as } n(\text { even }) \text { goes to infinity. }
$$

Remark. Unfortunately, the integral $m(n)$ cannot be $o\left(\frac{n}{\log ^{2} n}\right)$; since for almost all $n$, the integral is asymptotically $(S(n)-1)\left(\frac{n}{\log ^{2} n}\right)$ where $S(n)$ is the usual singular series.

We assume $n>C_{0}$. For each $n$ let $E_{n}=\left[x_{0}, 1-x_{0}\right]$.

Let $r(n)$ be the number of twin primes, each of which is less than $n$.

Clearly,

$$
r(n)=\int_{x_{0}}^{1+x_{0}}|f(x, n)|^{2} \in(-2 x) \mathrm{d} x
$$

We decompose this integral

$$
r(n)=m(n)+T(1,1)
$$

where

$$
r(n)=m(n)+T(1,1)
$$

where

$$
m(n)=\int_{E_{n}}|f(x, n)|^{2} \epsilon(-2 x) \mathrm{d} x, T(1,1)=\int_{1-x_{0}}^{1+x_{0}}|f(x, n)|^{2} \epsilon(-2 x) \mathrm{d} x .
$$

Immediately, from (3.3) we have

$$
\left.|| f(1+y, n)\right|^{2}-|g(y, n)|^{2}|\leq| f^{2}(1+y, n)-g^{2}(y, n) \mid \leq C_{2} n^{2} \log ^{-98+\epsilon} n .
$$

By the change of variable $y=(x-1)$, we have

$$
T(1,1)=\int_{-x_{0}}^{x_{0}}|f(1+y, n)|^{2} \epsilon(-2 y) \mathrm{d} y
$$

so that

$$
\left.\left|\int_{-x_{0}}^{x_{0}}\right| f(1+y, n)\right|^{2} \epsilon(-2 y) \mathrm{d} y-\int_{-x_{0}}^{x_{0}}|g(y, n)|^{2} \epsilon(-2 y) \mathrm{d} y\left|\leq \int_{-x_{0}}^{x_{0}}\right||f(1+y, n)|^{2}-|g(y, n)|^{2} \mid \mathrm{d} y<C_{6} n \log ^{-96+2 \epsilon} n .
$$

Let

$$
\begin{gathered}
T_{1}(n)=\int_{-x_{0}}^{x_{0}}|g(y, n)|^{2} \epsilon(-2 y) \mathrm{d} y . \\
\left|T(1,1)-T_{1}(n)\right| \leq C_{6} n \log ^{-96+2 \epsilon} n .
\end{gathered}
$$

Let

$$
T(n)=\sum_{\substack{m_{1} m_{2} \\ m_{1} \geq m_{2}, m_{2} \geq 2 \text { and } m_{2}-m_{1}=2 .}} \log ^{-1} m_{1} \log ^{-1} m_{2} .
$$


Clearly,

$$
T(n)=\int_{-\frac{1}{2}}^{\frac{1}{2}}|g(y, n)|^{2} \epsilon(-2 y) \mathrm{d} y .
$$

Also, the number of terms on the right hand side of (3.7) is $(n-3)$ and each term is greater than $\log ^{-2} n$ and less than 1 so that

$$
\frac{1}{3} n \log ^{-2} n<T(n)<n
$$

Hence by definition of $g(y, n)$ and Abel's lemma we have

$$
|g(y, n)|<|y|^{-1},\left(0<|y|<\frac{1}{2}\right)
$$

so that

$$
\left|T(n)-T_{1}(n)\right| \leq 2 \int_{x_{0}}^{\frac{1}{2}} y^{-2} \mathrm{~d} y \leq C_{4} n \log ^{-(2+\epsilon)} n .
$$

Hence

$$
|T(1,1)-T(n)| \leq C_{6} n \log ^{-96+2 \epsilon} n+C_{4} n \log ^{-(2+\epsilon)} n .
$$

Let $S(n)=1$. Then $|T(n) S(n)-T(n)|=0$; so that

$$
|r(n)-T(n) S(n)| \leq C_{7} n \log ^{-(2+\epsilon)} n+|m(n)|
$$

so that we have $r(n) \rightarrow \infty$ if $m(n)=o\left(n / \log ^{2} n\right)$ as $n$ goes to infinity in some suitable sequence.

\section{2) Part II}

For each (even) $n>C_{0}$ let $p_{n}$ be a prime in $\left[2\left(\log ^{\theta} n+\frac{1}{2}\right), 4\left(\log ^{\theta} n+\frac{1}{2}\right)\right]$. Let $E_{n}$ be those points in $\left[x_{0}, 1+x_{0}\right]$ which are not in $\left[1-x_{0}, 1+x_{0}\right]$ and not in any closed interval of radius $x_{0}$ about any rational number $\frac{h}{p_{n}}$ where

$$
\begin{aligned}
0< & <p_{n} . \\
\text { Let } m & =\left[\log ^{\theta_{1}} n\right] . \\
\text { Let } f_{1}(x, v) & =\sum_{\substack{p \leq v \\
p=1 \bmod p_{n} \\
p=2 \bmod p_{n} \\
p \equiv m \bmod p_{n}}} \epsilon(p x) .
\end{aligned}
$$

Let $r(n)$ be the number of representations of $n$ as the sum of two primes, which are limited to those primes in the $m$ arithmetic progressions mod $p_{n}$.

Clearly,

$$
r(n)=\int_{x_{0}}^{1+x_{0}} f_{1}^{2}(x, n) \epsilon(-n x) \mathrm{d} x .
$$

We decompose this integral

$$
r(n)=m(n)+T^{*}(1,1)+\sum_{0<h<p_{n}} T\left(h, p_{n}\right),
$$


where

$$
\begin{gathered}
m(n)=\int_{E_{n}} f_{1}^{2}(x, n) \epsilon(-n x) \mathrm{d} x, \\
T^{*}(1,1)=\int_{1-x_{0}}^{1+x_{0}} f_{1}^{2}(x, n) \epsilon(-n x) \mathrm{d} x, \\
T\left(h, p_{n}\right)=\int_{\frac{h}{p_{n}}-x_{0}}^{\frac{h}{p_{n}}+x_{0}} f_{1}^{2}(x, n) \epsilon(-n x) \mathrm{d} x,\left(0<h<p_{n}\right), \\
|m(n)| \leq \int_{x_{0}}^{1+x_{0}}\left|f_{1}(x, n)\right|^{2} \mathrm{~d} x \leq C_{8} \frac{m^{2} n}{\phi\left(p_{n}\right) \log n} \leq C_{9} \frac{n}{\log \left(\theta+1-2 \theta_{1}\right)} n \\
T^{*}(1,1)=T(1,1)+\int_{1-x_{0}}^{1+x_{0}}\left(f_{1}^{2}(x, n)-f^{2}(x, n)\right) \epsilon(-n x) \mathrm{d} x .
\end{gathered}
$$

\section{Conjecture 1.}

$$
I(n)=\int_{1-x_{0}}^{1+x_{0}}\left(f_{1}^{2}(x, n)-f^{2}(x, n)\right) \epsilon(-n x) \mathrm{d} x=o\left(\frac{n}{\log ^{2} n}\right) \text { as } n \text { (even) goes to infinity. }
$$

We now estimate $T\left(h, p_{n}\right), 0<h<p_{n}$.

$$
\begin{aligned}
f\left(\frac{h}{p_{n}}, v\right)= & \sum_{\substack{p \leq v \\
p \equiv 1 \bmod p_{n} \\
\vdots \\
p \equiv m \bmod p_{n}}} \epsilon\left(\frac{p h}{p_{n}}\right)=\sum_{\substack{p \leq v \\
p \equiv 1 \bmod p_{n}}} \epsilon\left(\frac{p h}{p_{n}}\right)+\cdots+\sum_{\substack{p \leq v \\
p \equiv m \bmod p_{n}}} \epsilon\left(\frac{p h}{p_{n}}\right) \\
& =\epsilon\left(\frac{h}{p_{v}}\right) \pi\left([v], p_{n}, 1\right)+\cdots+\epsilon\left(\frac{m h}{p_{n}}\right) \pi\left([v], p_{n}, m\right) .
\end{aligned}
$$

But consider

$$
\begin{gathered}
\epsilon\left(\frac{h}{p_{n}}\right) \pi\left([v], p_{v}, 1\right)-\epsilon\left(\frac{h}{p_{v}}\right) \frac{l s[v]}{\phi\left(p_{n}\right)} \\
\vdots \\
\epsilon\left(\frac{m h}{p_{n}}\right) \pi\left([v], p_{v}, m\right)-\epsilon\left(\frac{m h}{p_{v}}\right) \frac{l s[v]}{\phi\left(p_{v}\right)}
\end{gathered}
$$

so that uniformly $0 \leq v \leq n$

$$
\begin{gathered}
\left|\epsilon\left(\frac{h}{p_{n}}\right) \pi\left([v], p_{v}, 1\right)-\epsilon\left(\frac{h}{p_{v}}\right) \frac{l s[v]}{\phi\left(p_{v}\right)}\right| \leq\left|\pi\left([v], p_{v}, 1\right)-\frac{l s[v]}{\phi\left(p_{v}\right)}\right| \leq n \log ^{-100} n . \\
\vdots \\
\left|\epsilon\left(\frac{m h}{p_{n}}\right) \pi\left([v], p_{v}, m\right)-\epsilon\left(\frac{m h}{p_{n}}\right) \frac{l s[v]}{\phi\left(p_{v}\right)}\right| \leq\left|\pi\left([v], p_{v}, m\right)-\frac{l s[v]}{\phi\left(p_{v}\right)}\right| \leq n \log ^{-100} n .
\end{gathered}
$$

Hence for $0<h<p_{n}$ uniformly $0 \leq v \leq n$

$$
\left|f\left(\frac{h}{p_{n}}, v\right)-\left(\sum_{l=1}^{m} \epsilon\left(\frac{l h}{p_{n}}\right)\right) \frac{l s[v]}{\phi\left(p_{v}\right)}\right| \leq C_{10} m n \log ^{-100} n .
$$


So by (100) and (101) for $|y| \leq x_{0},\left(0<h<p_{n}\right)$

$$
\left|f\left(\frac{h}{p_{n}}+y, n\right)-\left(\sum_{l=1}^{m} \epsilon\left(\frac{l h}{p_{n}}\right)\right) \frac{g(y, n)}{\phi\left(p_{n}\right)}\right| \leq C_{11} m n \log ^{-98+\epsilon} n .
$$

But since $m<\left(p_{n}-1\right)=\phi\left(p_{n}\right),\left|\frac{\sum_{l=1}^{m} \epsilon\left(\frac{l h}{p_{n}}\right) g(y, n)}{\phi\left(p_{n}\right)}\right| \leq n$ and since $\left|f\left(\frac{h}{p_{n}}+y, n\right)\right| \leq n$, we have by the inequalities immediately below (3.2) for $|y| \leq x_{0},\left(0<h<p_{n}\right)$

$$
\left|f^{2}\left(\frac{h}{p_{n}}+y, n\right)-\left(\sum_{l=1}^{m} \epsilon\left(\frac{l h}{p_{n}}\right)\right)^{2} \frac{g^{2}(y, n)}{\phi^{2}\left(p_{n}\right)}\right| \leq C_{12} m n^{2} \log ^{-98+\epsilon} n .
$$

But

$$
\left|\left(\sum_{l=1}^{m} \in\left(\frac{l h}{p_{n}}\right)\right)^{2}\left(\frac{g^{2}(y, n)}{\phi^{2}\left(p_{n}\right)}\right)-\left(\frac{g^{2}(y, n)}{\phi^{2}\left(p_{n}\right)}\right)\right| \leq C_{13} m^{2} n^{2} \log ^{-2 \theta-2} n .
$$

Hence for $|y| \leq x_{0},\left(0<h<p_{n}\right)$

$$
\left|f^{2}\left(\frac{h}{p_{n}}+y, n\right)-\frac{g^{2}(y, n)}{\phi^{2}\left(p_{n}\right)}\right| \leq C_{12} m n^{2} \log ^{-98+\epsilon} n+C_{13} m^{2} n^{2} \log ^{-2 \theta-2} n .
$$

By a change of variable $y=\left(x-\frac{h}{p_{n}}\right)$ we have

$$
T\left(h, p_{n}\right)=\epsilon\left(-\frac{n h}{p_{n}}\right) \int_{-x_{0}}^{x_{0}} f^{2}\left(\frac{h}{p_{n}}+y, n\right) \epsilon(-n y) \mathrm{d} y .
$$

However,

$$
\begin{aligned}
& \left|\epsilon\left(-\frac{n h}{p_{n}}\right) \int_{-x_{0}}^{x_{0}} f^{2}\left(\frac{h}{p_{n}}+y, n\right) \epsilon(-n y) \mathrm{d} y-\frac{1}{\phi^{2}\left(p_{n}\right)} \epsilon\left(-\frac{n h}{p_{n}}\right) \int_{-x_{0}}^{x_{0}} g^{2}(y, n) \epsilon(-n y) \mathrm{d} y\right| \\
& \leq \int_{-x_{0}}^{x_{0}}\left|f^{2}\left(\frac{h}{p_{n}}+y, n\right)-\frac{g^{2}(y, n)}{\phi^{2}\left(p_{n}\right)}\right| \mathrm{d} y \leq C_{14} m n \log ^{-96+2 \epsilon} n+C_{15} m^{2} n \log ^{-2 \theta+\epsilon} n .
\end{aligned}
$$

Let

$$
T_{1}(n)=\int_{-x_{0}}^{x_{0}} g^{2}(y, n) \epsilon(-n y) \mathrm{d} y .
$$

Hence, if $0<h<p_{n}$,

$$
\begin{aligned}
& \left|T\left(h, p_{n}\right)-\frac{T_{1}(n)}{\phi^{2}\left(p_{n}\right)} \epsilon\left(-\frac{m h}{p_{n}}\right)\right| \leq C_{16} m^{2} n \log ^{-2 \theta+\epsilon} n . \\
& \text { Let } T(n)=\sum_{\substack{m_{1} \geq m_{2}, m_{2} \geq 2 \text { and } m_{1}+m_{2}=n \\
\log ^{-1} m_{1} \log ^{-1} m_{2} .}} \log .
\end{aligned}
$$

By (3.5) we have

$$
\left|T(n)-T_{1}(n)\right| \leq C_{4} n \log ^{-(2+\epsilon)} n,
$$

and 


$$
\frac{1}{3} n \log ^{-2} n<T(n)<n
$$

But

$$
\left|\epsilon\left(-\frac{n h}{p_{n}}\right)\left(\frac{1}{\phi^{2}\left(p_{n}\right)}\right)\right|\left|T(n)-T_{1}(n)\right| \leq C_{4} \frac{n \log ^{-(2+\epsilon)} n}{\phi^{2}\left(p_{n}\right)} .
$$

Hence, if $0<h<p_{n}$,

$$
\left|T\left(h, p_{n}\right)-\frac{T(n)}{\phi^{2}\left(p_{n}\right)} \epsilon\left(-\frac{n h}{p_{n}}\right)\right| \leq C_{16} m^{2} n \log ^{-2 \theta+\epsilon} n+C_{4} \frac{n \log ^{-(2+\epsilon)} n}{\phi^{2}\left(p_{n}\right)} ;
$$

so that

$$
\left|\sum_{0<h<p_{n}} T\left(h, p_{n}\right)-\frac{T(n)}{\phi^{2}\left(p_{n}\right)} C_{p_{n}}(n)\right| \leq C_{17} m^{2} n \log ^{-\theta+\epsilon} n+C_{4} \frac{n \log ^{-(2+\epsilon)} n}{\phi\left(p_{n}\right)}
$$

where

$$
C_{p_{n}}(n)=\sum_{0<h<p_{n}} \epsilon\left(-\frac{n h}{p_{n}}\right) .
$$

But by (3.6) we have

$$
|T(1,1)-T(n)| \leq C_{18} n \log ^{-(2+\epsilon)} n ;
$$

so that

$$
\left|\sum_{0<h<p_{n}} T\left(h, p_{n}\right)+T(1,1)-T(n)\left(1+\frac{C_{p_{n}}(n)}{\phi^{2}\left(p_{n}\right)}\right)\right| \leq C_{19} n \log ^{-\theta+2 \theta_{1}+\epsilon} n ;
$$

so that

$$
\left|r(n)-T(n)\left(\frac{1+C_{p_{n}}(n)}{\phi^{2}\left(p_{n}\right)}\right)\right| \leq C_{19} n \log ^{-\theta+2 \theta_{1}+\epsilon} n+|I(n)|+|m(n)| .
$$

Let

$$
S(n)=\left(1+\frac{C_{p_{n}}(n)}{\phi^{2}\left(p_{n}\right)}\right)
$$

By Theorem 272 in [5]

so that $S(n)>\frac{1}{2}$, since we assume $n>C_{0}$.
Hence

$$
C_{p_{n}}(n)=\left\{\begin{array}{l}
-1 \text { if }\left(n, p_{n}\right)=1 \\
\left(p_{n}-1\right) \text { if }\left(n, p_{n}\right)>1
\end{array}\right.
$$

$$
|r(n)-T(n) S(n)| \leq C_{19} n \log ^{-\theta+2 \theta_{1}+\epsilon} n+|I(n)|+|m(n)|
$$

so that $r(n)>0$ if Conjecture 1 is true.

Let $r(n)$ be the number of twin primes, each of which is in one of the arithmetic progressions mod $p_{n}$ defined above.

Clearly,

$$
r(n)=\int_{X_{0}}^{1+x_{0}}\left|f_{1}(x, n)\right|^{2} \epsilon(-2 x) \mathrm{d} x
$$


We decompose the integral

$$
\begin{aligned}
& r(n)=m(n)+T^{*}(1,1)+\sum_{0<h<p_{n}} T\left(h, p_{n}\right), \text { where } \\
& m(n)=\int_{E_{n}}\left|f_{1}(x, n)\right|^{2} \epsilon(-2 x) \mathrm{d} x, \\
& T^{*}(1,1)=\int_{1-x_{0}}^{1+x_{0}}\left|f_{1}(x, n)\right|^{2} \epsilon(-2 x) \mathrm{d} x, \\
& T\left(h, p_{n}\right)=\int_{\frac{h}{p_{n}}}^{\frac{h}{p_{n}}+x_{0}}\left|f_{1}(x, n)\right|^{2} \epsilon(-2 x) \mathrm{d} x, \\
& T^{*}(1,1)=T(1,1)+\int_{1-x_{0}}^{1+x_{0}}\left(\left|f_{1}(x, n)\right|^{2}-|f(x, n)|^{2}\right) \epsilon(-2 x) \mathrm{d} x .
\end{aligned}
$$

\section{Conjecture 2.}

$$
I(n)=\int_{1-x_{0}}^{1+x_{0}}\left(\left|f_{1}(x, n)\right|^{2}-|f(x, n)|^{2}\right) \epsilon(-2 x) \mathrm{d} x=o\left(\frac{n}{\log ^{2} n}\right) \text {, as } n \text { goes to }
$$

infinity in some suitable sequence.

From (3.10) we have for $|y| \leq x_{0},\left(0<h<p_{n}\right)$

$$
\left|f\left(\frac{h}{p_{n}}+y, n\right)\right|^{2}-\frac{|g(y, n)|^{2}}{\phi^{2}\left(p_{n}\right)} \mid \leq C_{20} m^{2} n^{2} \log ^{-2 \theta-2} n .
$$

By change of variable $y=\left(x-\frac{h}{p_{n}}\right)$ we have

$$
T(h, q)=\epsilon\left(\frac{-2 h}{p_{n}}\right) \int_{-x_{0}}^{x_{0}}\left|f^{2}\left(\frac{h}{p_{n}}+y, n\right)\right|^{2} \epsilon(-2 y) \mathrm{d} y .
$$

However,

$$
\begin{aligned}
& \left.\left|\epsilon\left(\frac{-2 h}{n}\right) \int_{-x_{0}}^{x_{0}}\right| f\left(\frac{h}{p_{n}}+y, n\right)\right|^{2} \in(-2 y)-\frac{1}{\phi^{2}\left(p_{n}\right)} \epsilon\left(\frac{-2 h}{n}\right) \int_{-x_{0}}^{x_{0}}|g(y, n)|^{2} \epsilon(-2 y) \mathrm{d} y \mid \\
& \leq \int_{-x_{0}}^{x_{0}}\left|f\left(\frac{h}{p_{n}}+y, n\right)\right|^{2}-\frac{|g(y, n)|^{2}}{\phi^{2}\left(p_{n}\right)} \mid \mathrm{d} y \leq C_{21} m^{2} n \log ^{-2 \theta+\epsilon} n .
\end{aligned}
$$

Let

$$
T_{1}(n)=\int_{-x_{0}}^{x_{0}}|g(y, n)|^{2} \epsilon(-2 y) \mathrm{d} y
$$

Hence, if $0<h<p_{n}$

$$
\begin{aligned}
& \left|T\left(h, p_{n}\right)-\frac{T_{1}(n)}{\phi^{2}\left(p_{n}\right)} \epsilon\left(-\frac{2 h}{p_{n}}\right)\right| \leq C_{21} m^{2} n \log ^{-2 \theta+\epsilon} n . \\
& \text { Let } T(n)=\sum_{\substack{m_{1}, m_{2} \\
m_{1} \geq 2, m_{2} \geq 2 \text { and } m_{2}-m_{1}=2}} \log ^{-1} m_{1}, \log ^{-1} m_{2} .
\end{aligned}
$$

Clearly, 


$$
T(n)=\int_{-\frac{1}{2}}^{\frac{1}{2}}|g(y, n)|^{2} \epsilon(-2 y) \mathrm{d} y
$$

So that by (3.8)

$$
\left|T(n)-T_{1}(n)\right| \leq C_{4} n \log ^{-(2+\epsilon)} n
$$

and $\frac{1}{3} n \log ^{-2} n<T(n)<n$.

$$
\left|\epsilon\left(-\frac{2 h}{p_{n}}\right)\left(\frac{1}{\phi^{2}\left(p_{n}\right)}\right)\right| T(n)-T_{1}(n) \mid \leq C_{4} \frac{n \log ^{-(2+\epsilon)} n}{\phi^{2}\left(p_{n}\right)} .
$$

Hence, if $0<h<p_{n}$,

$$
\left|T\left(h, p_{n}\right)-\frac{T(n)}{\phi^{2}\left(p_{n}\right)} \epsilon\left(-\frac{2 h}{p_{n}}\right)\right| \leq C_{21} m^{2} n \log ^{-2 \theta+\epsilon} n+C_{4} \frac{n \log ^{-(2+\epsilon)} n}{\phi^{2}\left(p_{n}\right)} ;
$$

so that

$$
\left|\sum_{0<h<p_{n}} T\left(h, p_{n}\right)-\frac{T(n)}{\phi^{2}\left(p_{n}\right)} C_{p_{n}}(2)\right| \leq C_{22} \log ^{-\theta+2 \theta_{1}+\epsilon} n+C_{4} \frac{n \log ^{-(2+\epsilon)} n}{\phi\left(p_{n}\right)}
$$

where

$$
C_{p_{n}}(2)=\sum_{0<h<p_{n}} \epsilon\left(-\frac{2 h}{p_{n}}\right) .
$$

But by (3.6)

$$
|T(1,1)-T(n)| \leq C_{4} n \log ^{-(2+\epsilon)} n
$$

so that

$$
\left|\sum_{0<h<p_{n}} T\left(h, p_{n}\right)+T(1,1)-T(n)\left(1+\frac{C_{p_{n}}(2)}{\phi^{2}\left(p_{n}\right)}\right)\right| \leq C_{22} n \log ^{-\theta+2 \theta_{1}+\epsilon} n .
$$

Hence

$$
\begin{gathered}
\left|r(n)-T(n)\left(1+\frac{C_{p_{n}}(2)}{\phi^{2}\left(p_{n}\right)}\right)\right| \leq C_{22} n \log ^{-\theta+2 \theta_{1}+\epsilon} n+|I(n)|+|m(n)| . \\
\text { Let } S(n)=\left(1+\frac{C_{p_{n}}(2)}{\phi^{2}\left(p_{n}\right)}\right) . S(n)>\frac{1}{2} \text { if } n>C_{0} .
\end{gathered}
$$

Hence

$$
|r(n)-T(n) S(n)| \leq C_{22} n \log ^{-\theta+2 \theta_{1}+\epsilon} n+|I(n)|+|m(n)|
$$

so that $r(n) \rightarrow \infty$; if Conjecture 2 is true, as $n$ goes to infinity in a sequence that satisfies the conjecture.

\section{Some Heuristics}

\section{Theorem 4.1 If}

$$
\int_{\left[-\left(2+x_{0}\right),\left(2+x_{0}\right)\right]-M(n)} f^{2}(x, n) \epsilon(-n x) \mathrm{d} x=o\left(\frac{n}{\log ^{2} n}\right),
$$

then every sufficiently large even integer is the sum of two primes, where $M(n)$ is an exceptional set, whose measure goes to 0 with $n$.

Proof. This is established in [1]. 
Theorem 4.2 Let $m$ be an arbitrary fixed integer. Then

$$
\int_{-2}^{2} \frac{f^{2}(x, m) x \epsilon(-n x)}{x-t} \mathrm{~d} x=o(\log \log n)
$$

uniformly for almost all $t \in(-1 / 2,1 / 2)$.

Proof. This deep result is immediate by (5-2) in [6].

There is no compelling reason to assume Theorem 4.2 is not true for $t=0$.

It is worthwile to investigate if Carleson's proof can be modified to establish Theorem 4.2 with $\mathrm{m}$ replaced with $n$ and $o(\log \log n)$ replaced with $o\left(\frac{n}{\log ^{2} n}\right)$.

In [7] Tao presents a heuristic argument to establish that the major arc contribution in the circle method is $o\left(\frac{n}{\log ^{2} n}\right)$. He states that his argument can be made rigorous. However, it follows from the proof of Theorem 1.5 that the major arc contribution is not $o\left(\frac{n}{\log ^{2} n}\right)$ in any
sequence of $n$.

But it is well known that $r^{\prime}(n)=0\left(\frac{n}{\log ^{2} n}\right)$ so that the contribution of the minor arc in the circle method approach to the twin primes conjecture (Theorem 1.5) is $o\left(\frac{n}{\log ^{2} n}\right)$, which makes plausible that the required estimate of $o\left(\frac{n}{\log ^{2} n}\right)$ might be true.

It is plausible that in Theorem 1.3

$$
\int_{m(n)} f^{2}(x, m) \epsilon(-m x) \mathrm{d} x=0\left(\int_{m(n)}|f(x, m)|^{2} \epsilon(-2 x) \mathrm{d} x\right)
$$
where the latter integral is that of Theorem 1.5, which makes plausible that the required estimate of $o\left(\frac{n}{\log ^{2} n}\right)$
might be true.

Those, who seriously attempt Conjecture 2 have the advantage that there is some degree of freedom in the choice of $m=\left[\log ^{\theta_{1}} n\right]$ and in the choice of $p_{n}$ for each $n$; and the $o\left(n \log ^{-2} n\right)$ estimate is required only as $n$ goes to infinity in some suitable sequence.

\section{Acknowledgements}

I thank R. C. Vaughan for the Remark in Part I, Section 3.

\section{References}

[1] Mozzochi, C.J. and Balasubramanian, R. (1978) Some Comments on Goldbach’s Conjecture. Report No. 11, MittagLeffler Institute.

[2] Balasubramanian, R. and Mozzochi, C.J. (1983) Siegel Zeros and the Goldbach Problem. Journal of Number Theory, 16, 311-332.

[3] Estermann, T. (1961) Introduction to Modern Prime Number Theory. Cambridge Univ. Press, London/New York.

[4] Montgomery, H.L. and Vaughan, R.C. (1973) Error Terms in Additive Prime Number Theory. Quarterly Journal of Mathematics, 24, 207-216.

[5] Hardy, G.H. and Wright, E.M. (1965) An Introduction to the Theory of Numbers. 4th Edition, Oxford University Press, London/New York.

[6] Carleson, L. (1966-1967) Sur la convergence et l'order des gradeur des somes partielles des series de Fourier. Marseille Notes.

[7] Tao, T. (2012) Heuristic Limitations of the Circle Method. Blog Post 20 May 2012, 1-11. 